\title{
Das Onlineangebot der AO Trauma Deutschland
}

Karl-Friedrich Braun, Ulrich Stöckle

Die notwendigen Schritte zur Eindämmung der Pandemie stellen uns alle vor große berufliche und private Herausforderungen. Gleichzeitig haben genau diese Veränderungen zu einer raschen digitalen Transformation in allen Lebensbereichen geführt und unter anderem die Digitalisierung der Medizin vorangetrieben. Onlinesprechstunden, Klinikkonferenzen auf Skype und weitere „kontaktarme oder -lose“ Angebote haben Einzug in unseren Klinikalltag gehalten. Die Einführung und Auseinandersetzung mit in- novativer Technologie und ihren Möglichkeiten ist kernentscheidend für die Zeit während und nach Social Distancing. Gleiches gilt für die Wissensvermittlung - hier wurde vielen Universitäten und Instituten ihr dringender Nachholbedarf in digitaler Ausbildung klar vor Augen geführt.

Auch in der deutschen AO mussten zunächst alle Kurse auf unbestimmte Zeit ausgesetzt werden. Dies wurde auch als Chance gesehen, an alternativen Lehrformaten
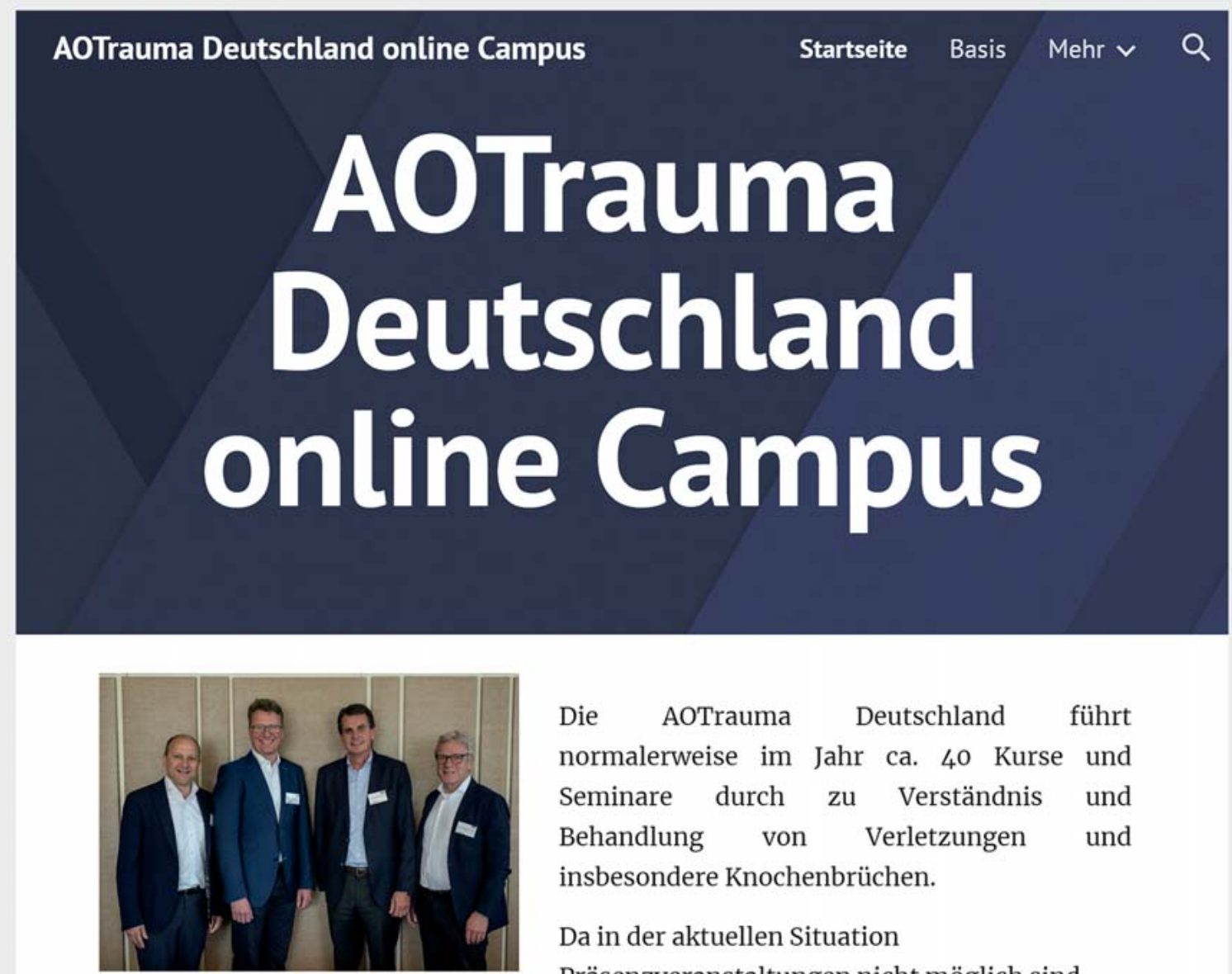

Die AOTrauma Deutschland führt normalerweise im Jahr ca. 40 Kurse und Seminare durch zu Verständnis und Behandlung von Verletzungen und insbesondere Knochenbrüchen.

Da in der aktuellen Situation

Präsenzveranstaltungen nicht möglich sind, haben wir diesen „online Campus" gegründet, um ein webbasiertes Schulungsangebot aufzubauen und dauerhaft zu etablieren.

- Abb. 1 Website für den „online Campus“. 


\section{Ablauf des Seminars}

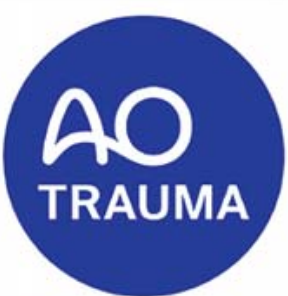

Dienstag

12. Mai 2020

17:00-18:00 Uhr

Einführung

Prof. Dr. Ulrich Stöckle, Berlin

Vorstellung AO

Prof. Dr. Florian Gebhard, Ulm

Prinzipien der Knochenheilung

Prof. Dr. Joachim Windolf,

Düsseldorf
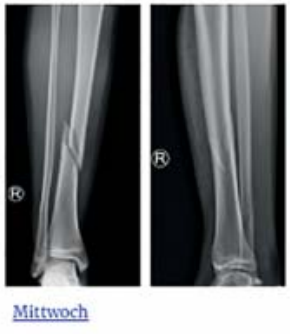

13. Mai 2020

17:00-18:00 Uhr

Frakturen Tibiaschaft -

Prinziplen der Versorgung

Fixateur, Marknagel, Platte

Prof. Dr. Phillipp Kobbe, Aachen

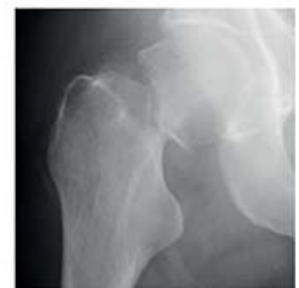

Montag

18. Mai 2020

17:00-18:00 thr

Junges Forum U\&O

Dr. Annika Hattich

Schenkelhalsfrakturen

Prof. Dr. Christor Burger, Bonn

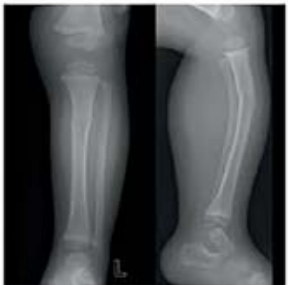

Mittwoch

20. Mai 2020

17:00-18:00 Uhr

Neustart nach Corona PD Dr. Andrej Trampuz

Prinzipien der Behandlung kindlicher Frakturen Prof. Dr. Tìm Lögters, Köln

- Abb. 2 Screenshot des Mai-Angebots im Onlinecampus.

zu arbeiten. Im April wurde das Pilotprojekt des „online Campus“ ( $\triangleright$ Abb. 1) initiiert (mit Unterstützung der Lohmann \& Birkner $\mathrm{GmbH}$ ) - zunächst als kostenloser Ersatz für die nicht stattfindenden Trauma-Basiskurse ( $\vee$ Abb. 2). Dabei wird den ursprünglichen Teilnehmern ein „Online-Seminar Basis“ angeboten mit 4 Terminen à $1 \mathrm{~h}$ und begrenzter Teilnehmerzahl. Dabei werden anteilig Inhalte des ursprünglichen Kurses abgedeckt und auch zusätzliche Themen besprochen wie z.B. „Normalisierung unter Corona“.

Das Projekt folgt neben einem strukturierten Live-Vortragskonzept hochrangiger Experten auch einem modernen „banana principle“. Dies ermöglicht die interaktive Verbesserung des Lehrformats durch unmittelbares Feedback der Teilnehmer in Kombination mit einem hohen Maß an experimentellen digitalen Arbeitsmethoden. Als Beispiel sei hier die Nutzung eines sogenannten „Jamboards“ erwähnt, in denen die Teilnehmer in Kleingruppen online klinische Fälle gemeinsam lösen und interaktiv nach Therapieplänen und -lösungen suchen. Dies ermöglicht, im Anschluss an die Vorträge das erlernte Wissen zu festigen und im Gespräch mit den Experten zu vertiefen.

Bisher sind 2 „Online-Seminare Basis“ erfolgt, für Juni geplant ist ein „Online-Seminar ORP“ sowie ein Online-Se- minar „Tibiakopf“ und eines zum Thema „Becken“. Mit den „Online-Vorträgen“ wird eine Bibliothek erstellt, deren Inhalt dann für spätere Kurse genutzt werden kann, als Ersatz oder Ergänzung von Präsenzveranstaltungen.

Der Aufbau eines Onlinecampus stellt somit die digitale Notwendigkeit im Wissenstransfer dar und bietet durch ein kontinuierlich angestrebtes Einbinden neuer Technologien eine Möglichkeit angewandter Lehrinnovation. Klar ist aber auch, dass wir alle uns auf die Wiederaufnahme der „Hands-on Kurse“ freuen - wir wissen aber auch, dass diese Kursformate bis auf Weiteres nicht in der herkömmlichen Form stattfinden können. Daher werden die entwickelten Onlineformate zumindest eine notwendige Ergänzung für die Zukunft darstellen.

PD Dr. med. Karl-Friedrich Braun

Prof. Dr. Ulrich Stöckle

Charité - Universitätsmedizin Berlin

Centrum für Muskuloskeletale Chirurgie

Bibliografie

DOI https://doi.org/10.1055/a-1200-3945

OP-JOURNAL 2020; 36: 168-169 @ Georg Thieme Verlag KG Stuttgart · New York ISSN 0178-1715 